\title{
AtomAI: Open-source software for applications of deep learning to microscopy data
}

\author{
Maxim Ziatdinov ${ }^{1}$ and Sergei Kalinin ${ }^{2}$
}

${ }^{1}$ Computational Sciences and Engineering Division, Oak Ridge National Laboratory, Oak Ridge, Tennessee, United States, ${ }^{2}$ Center for Nanophase Materials Sciences, Oak Ridge National Laboratory, Oak Ridge, Tennessee, United States

Development of the imaging tools such as scanning transmission electron and probe microscopies (STEM and SPM) in the last decade of the 20th century opened floodgates of imaging data, in the form of images, movies, and hyperspectral data sets. They contain information on the minute details of atomic structure and electronic, magnetic, and phonon functionalities, chemical transformation mechanisms, and quantum phenomena. The bottleneck in analysis and knowledge extraction is often a human domain expert. In the last several years the deep machine learning was shown to be an effective image analysis tool; however, the number of implemented algorithms for deep learning-based (quantitative) physics extraction from noisy experimental data is very limited.

AtomAI is an open-source (https://github.com/pycroscopy/atomai) Python library based on the Pytorch deep learning framework, whose goal is to provide a bridge environment between the instrument-specific libraries and general physical analysis by enabling the seamless deployment of the deep and machine learning algorithms to images and hyperspectral data. It has a simple and intuitive (scikit-learn-like) API and does not require any advanced knowledge of Python or machine learning to use it. Some of the basic functionalities of the AtomAI package include deep convolutional neural networks for semantic segmentation and atom/defect/particle detection, invariant variational autoencoders for finding in the unsupervised fashion the most effective reduced representation of the system's local descriptors ("building blocks"), Im2Spec and Spec2Im models for predicting spectra from local structural images and vice versa, ensemble learning for more accurate and reliable predictions with the quantified uncertainty, and multivariate statistical and graph analysis tools that can be applied to the outputs of the deep learning models for more in-depth physics exploration.

Below we show a simple example of using AtomAI for atom finding via atomic-level semantic segmentation of noisy experimental data. You can run this example by yourself in Google Colab using the link in Ref [1]. First, we train a deep convolutional neural network, which requires just two lines of code:

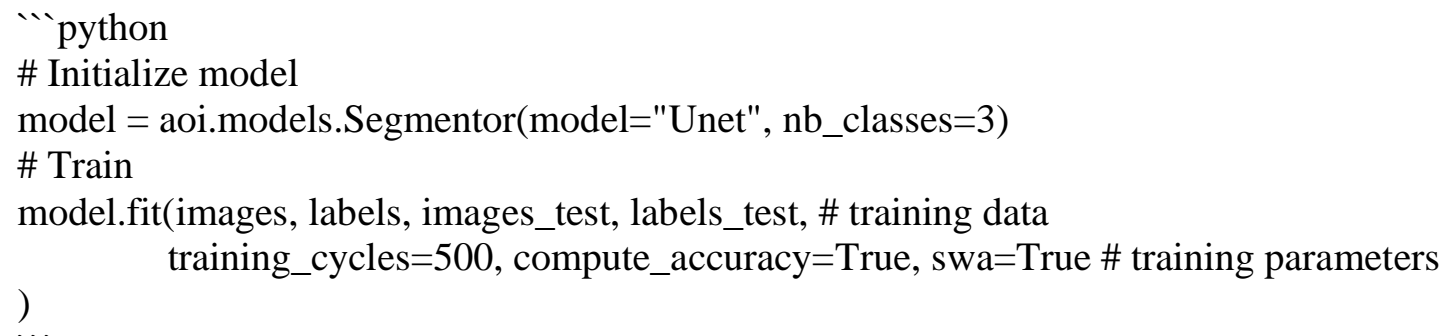

Here, swa stands for stochastic weight averaging, which allows improving the model's accuracy and leads to better generalization on noisy data. The trained model can be used to find atoms in new, previously unseen (by a model) data:

“'python

\# Get the semantically-segmented output and atomic coordinates

nn_output, coordinates $=$ model.predict(expdata) 
This operation takes about a millisecond on a modern GPU for an image of the 1024 px x 1024 px. Here, the nn_output is a semantically segmented output of the trained neural network, whereas the coordinates is a $N \mathrm{x}$ 3 array (or a dictionary of arrays, if the dataset is a stack of multiple images), where the first two dimensions are $x$ and $y$ coordinates and the third dimension is the atomic class. Please visit AtomAI's homepage on GitHub for more functionalities and examples including unsupervised deep learning with variational autoencoders and image $\rightarrow$ spectrum predictions.

In summary, AtomAI introduces easy-to-use deep/machine learning methods tailored for applications in scientific imaging. It combines the power and flexibility of the PyTorch deep learning framework with the simplicity and intuitive nature of packages such as the scikit-learn to ensure that there is a minimal-to-no barrier to start using it.

Target audience
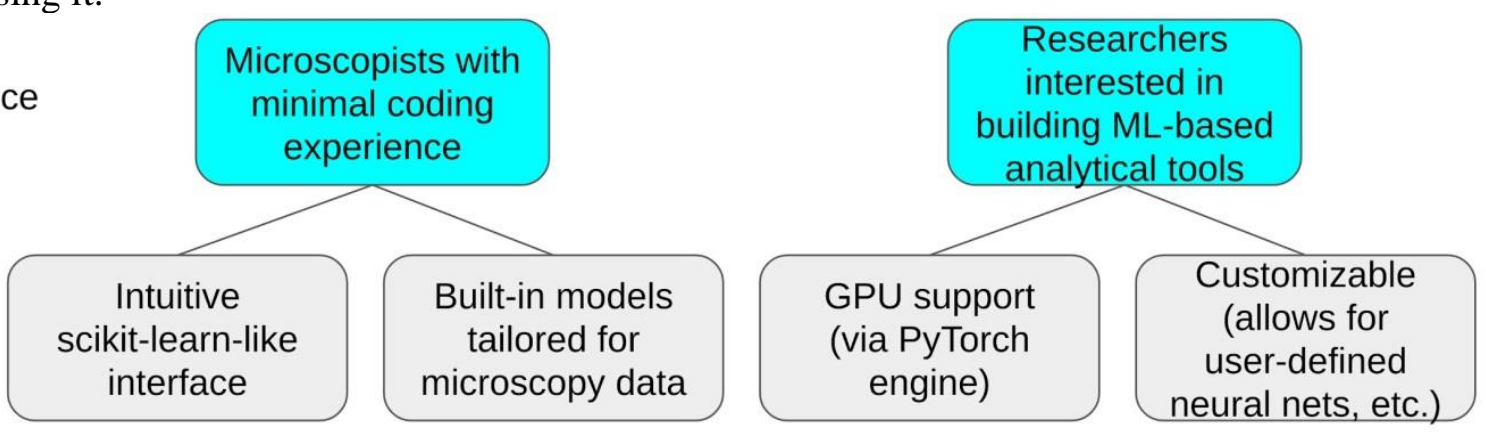

Figure 1. Figure 1. High-level schematics of AtomAI's features and the targeted audience.
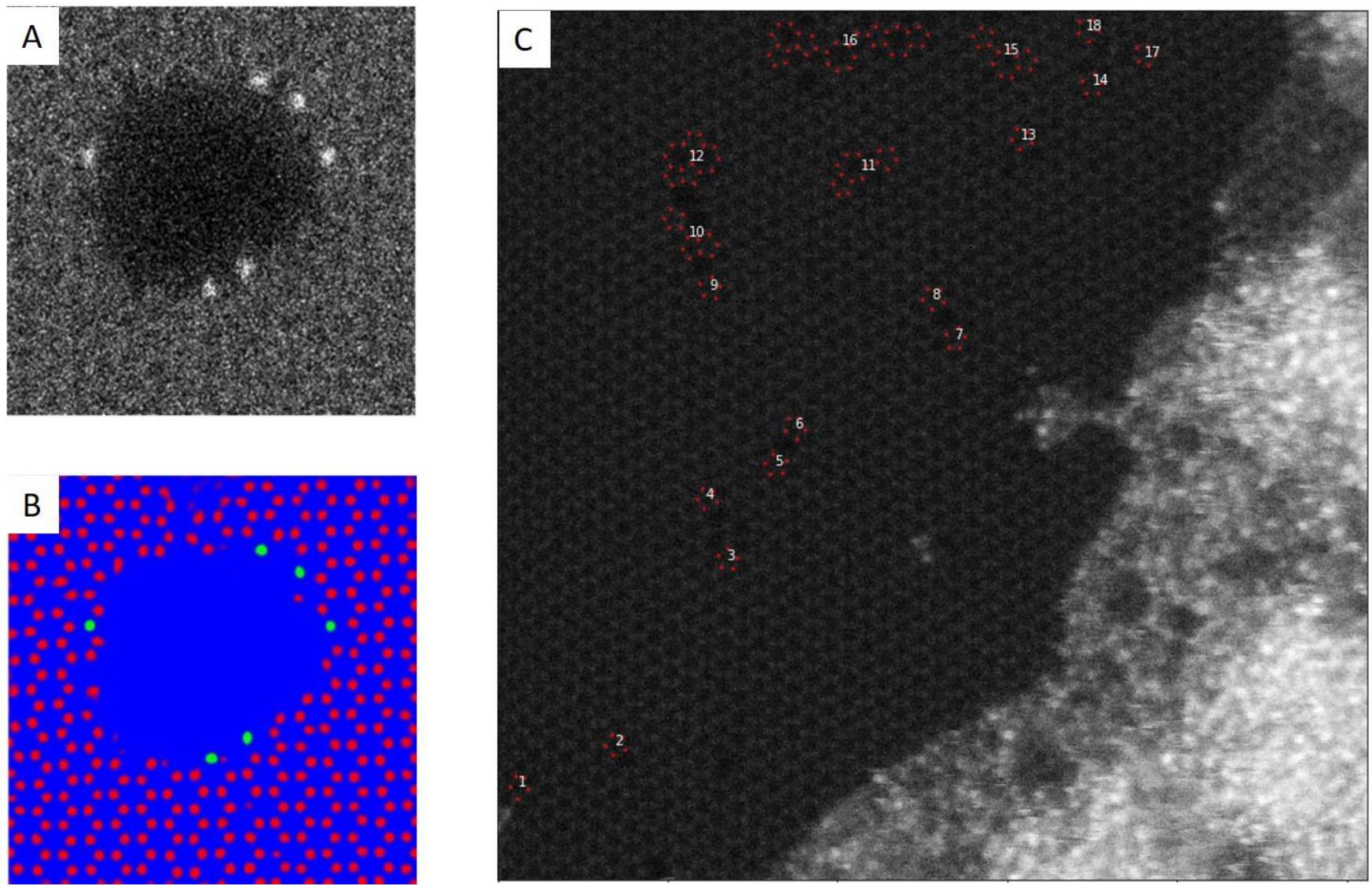

Figure 2. Figure 2. $(a, b)$ Example of the application of AtomAI'sSegmentor to noisy experimental data from graphene (c) Automated identification of graphene topological defects (containing 5- and/or 7-member rings) by applying graph analysis to the output of the semantic segmentation model. 


\section{References}

[1] Executable Google Colab notebook with the example discussed in the text: https://git.io/JtFJw [2] This effort was performed and supported at Oak Ridge National Laboratory's Center for Nanophase Materials Sciences (CNMS), a U.S. Department of Energy, Office of Science User Facility. 